\title{
Analysis of Information Flow in MISO Neuro-spike Communication Channel with Synaptic Plasticity
}

\author{
T. Khan ${ }^{1}$, H. Ramezani ${ }^{2}$, G. Muzio ${ }^{3}$ and O. B. Akan ${ }^{1,2}$ \\ ${ }^{1}$ Deparment of Electrical and Electronics Engineering, Koç University, 34450 Istanbul, Turkey \\ ${ }^{2}$ Internet of Everything (IoE) Group, Department of Engineering, University of Cambridge, CB3 OFA Cambridge, UK \\ ${ }^{3}$ Department of Electrical, Computer and Biomedical Engineering, University of Pavia, 27100 Pavia, Italy \\ e-mails: tkhan15@ku.edu.tr,hr404@cam.ac.uk, giulia.muzio01@universitadipavia.it,oba21@cam.ac.uk
}

\begin{abstract}
Communication among neurons is the most promising technique for biocompatible nanonetworks. This necessitates the thorough communication theoretical analysis of information transmission among neurons. The information flow in neuro-spike communication channel is regulated by the ability of neurons to change their synaptic strengths over time, i.e. synaptic plasticity. Thus, the performance evaluation of the nervous nanonetwork is incomplete without considering the influence of synaptic plasticity. Hence, in this paper, we provide a comprehensive model for multiple-input singleoutput (MISO) neuro-spike communication by integrating the spike timing dependent plasticity (STDP) into existing channel model. We simulate this model for a realistic scenario with correlated inputs and varying spiking threshold. We show that plasticity is strengthening the correlated input synapses at the expense of weakening the synapses with uncorrelated inputs. Moreover, a nonlinear behavior in signal transmission is observed with changing spiking threshold.
\end{abstract}

\section{INTRODUCTION}

Synaptic plasticity is a fundamental property of the mammalian brain and refers to the capability of synapses to change their strength [1]. It is involved in the control of information flow between neurons on different temporal scales and can be divided into two main categories, (i) short-term plasticity, which lasts on the order of milliseconds to several minutes [2], and (ii) long-term plasticity, which refers to changes that can last from hours to the lifetime of the synapse and is thought to play a central role in the mechanisms involved in memory and learning [1]. Moreover, aberrant synaptic plasticity contributes to several neuropsychiatric disorders [1], [3]. Hence, studying synaptic plasticity in both healthy and diseased conditions is an important step towards understanding brain functions and the development of novel diagnostic and treatment techniques.

Neuro-spike communication is one of the most promising nanoscale communication paradigms. Hence, several studies have focused on modeling processes involved and evaluating its performance [4]-[8]. However, while the short-term plasticity is considered in [4], [8], none of the existing studies in the literature have considered the impact of long-term synaptic plasticity on the performance of this communication channel. Our main motivation in this work is to study learning and memory processes that are based on the changes in strength and connectivity of neural networks. Hence, in this paper, for the first time in the literature, we study the impact of the long- term synaptic plasticity on multiple access communication channel among hippocampal-cortical neurons. Long-term plasticity can be expressed in two forms, (i) long-term potentiation (LTP), which refers to an enhancement in the strength of the synapse, and (ii) long-term depression (LTD), in which the synaptic strength is decreased over time [1]. Thus, in this paper, we are using bidirectional spike timing dependent plasticity (STDP) model that either depresses or strengthens the synapses depending on the temporal correlation between preand post-synaptic spikes [9].

Learning occurs through cooperation between synaptic inputs and the plasticity rules select inputs which are correlated with other inputs [10]. Thus, we analyze the influence of correlation among pre-synaptic spike trains on the strengthening of synapses and its impacts on the signal transmission over this channel. Moreover, impacts of variation in spiking threshold, which happens in real scenarios [10], is studied on the probability of spike generation in output neuron.

The remainder of this paper is organized as follows. In Section II, the MISO neuro-spike communication channel model is explained. Then, STDP and its impact on the synaptic channel strength is explained in Section III. Finally, the performance evaluation is provided in Section IV and the paper is concluded in Section V.

\section{MISO NeUro-SPIKE COMMUNICATION CHANNEL}

This study is based on a MISO synaptic channel, where a single post-synaptic neuron receives and processes information from multiple pre-synaptic neurons as shown in Fig. 1.

\section{A. Input spike trains and axonal transmission}

The synaptic communication begins with spike arrival in pre-synaptic neurons. The spike train in $i$ th pre-synaptic terminal can be modeled by Poisson process as $S_{i}(t)=$ $\operatorname{Poiss}\left(\lambda_{i}\right)$, where $\lambda_{i}$ is the spike rate, $i \in[1, M]$ and $M$ is the number of pre-synaptic neurons [5]. By discretizing time into windows of equal length, i.e., $\Delta t$, the spiking probability in $i$ th pre-synaptic neuron at $n$th time step is modeled as $P\left\{S_{i}[n]=\right.$ $1\}=1-\exp \left(-\lambda_{i} \Delta t\right)$, where $S_{i}[n]=1$ indicates spike arrival [4]. Moreover, after firing one spike, the neuron is not able to generate another spike for a certain time duration called refractory period, $\tau_{\text {ref }}[11]$. Hence, $P\left\{S_{i}[n]=1\right\}=0$ for $\frac{\tau_{r e f}}{\Delta t}$ consecutive time steps after firing one spike. Furthermore, since spikes propagate reliably through axons in hippocampal neurons [6], we model axonal transmission as ideal all pass filter with zero transmission delay. 


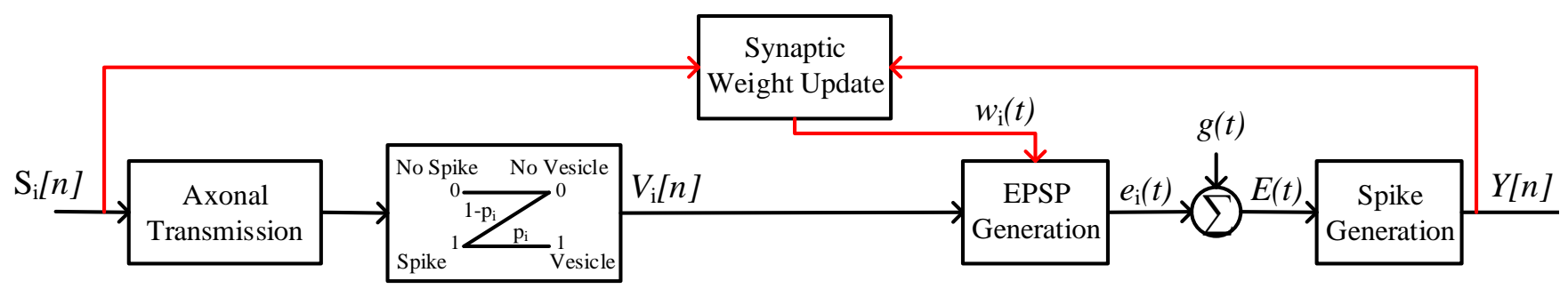

$i$ th Pre-synaptic Neuron

Post-synaptic Neuron

Fig. 1. Communication among one pre-synaptic neuron and the post-synaptic neuron in MISO synaptic channel.

\section{B. Vesicle Release Process}

The spike arrival at each pre-synaptic terminal causes vesicle release with probability $p_{i}$ modeled in [5] as follows,

$$
P\left\{V_{i}[n]=1 \mid S_{i}[n]=1\right\}=1-\exp \left(-\alpha_{i} N_{i}\right) \triangleq p_{i},
$$

where $\alpha_{i}=0.06 \sqrt{N_{i}}$ is the rate at which vesicles are fused with the membrane to get released [12], $N_{i}$ is the number of available vesicles for release and $V_{i}[n]=1$ indicate the vesicle release from $i$ th pre-synaptic terminal.

\section{Post-synaptic Response at Each Synapse}

After each release, the post-synaptic potential caused by $i$ th synapse is modeled in [5] as

$$
e_{i}(t)=w_{i}(t) h_{i} \frac{t}{t_{p}} \exp \left(1-\frac{t}{t_{p}}\right),
$$

where $t_{p}$ is the time to reach the peak amplitude of EPSP, $w_{i}(t) h_{i}$ is the peak EPSP amplitude at $i$ th synapse and $w_{i}(t)$ is the synaptic weight, which can increase or decrease according to the synaptic activity.

\section{Spike Generation}

The total membrane potential $E(t)$ at post-synaptic neuron contributed by all the inputs is given as

$$
E(t)=v_{\text {rest }}+\sum_{i=1}^{M} \sum_{\forall n: t_{n} \leq t} V_{i}[n] e_{i}\left(t-t_{n}\right)+g(t)
$$

where $v_{\text {rest }}$ is the membrane resting potential, $n$th time window starts at $t_{n}$ and $g(t)$ is the synaptic noise modeled by Gaussian distribution with zero mean and variance $\sigma_{n}^{2}$ [5]. A spike occurs in the output, i.e., $Y[n]=1$, when $E(t) \geq \theta$, where $\theta$ is the spiking threshold and $(n-1) \Delta t \leq t<n \Delta t$.

\section{SPIKe Timing Dependent Plasticity}

Strong depolarization of post-synaptic neuron is required for induction of LTP while weaker depolarization leads to LTD. Therefore, the change in synaptic efficacy is driven by temporal correlations between pre-synaptic spike arrival and postsynaptic firing and it is termed as STDP. STDP is order dependent, i.e., if the pre-synaptic spike arrives before postsynaptic spike, the synapse is strengthened, while the opposite order weakens the synapse. This kind of long-term synaptic plasticity is prevalent in cortical neurons especially in excitatory hippocampal pyramidal neurons [13]. Hence, in this section, we provide the model for updating the synaptic weights, governed by the variable $w_{i}(t)$, using STDP model.
Considering $S_{i}(t)$ and $Y(t)$ as pre- and post-synaptic spike trains, respectively, the change in weight of $i$ th synapse is given as,

$$
\begin{aligned}
\frac{d w_{i}(t)}{d t}= & a_{0}+S_{i}(t)\left(a_{1}^{\text {pre }}+\int_{0}^{\infty} a_{2}^{\text {pre } \text { post }}\left(t^{\prime}\right) Y\left(t-t^{\prime}\right) d t^{\prime}\right) \\
& +Y(t)\left(a_{1}^{\text {post }}+\int_{0}^{\infty} a_{2}^{\text {post }, \text { pre }}\left(t^{\prime}\right) S_{i}\left(t-t^{\prime}\right) d t^{\prime}\right),
\end{aligned}
$$

where $S_{i}(t)=\sum_{f} \delta\left(t-t_{i, p r e}^{f}\right), Y(t)=\sum_{f} \delta\left(t-t_{\text {post }}^{f}\right)$ and $t^{\prime}=t_{i, p r e}^{f}-t_{\text {post }}^{f}$ is the time difference between pre- and postsynaptic spikes. The parameter $a_{0}<0$ represents an activity independent constant decrease in the synaptic weight, i.e., irrespective of the presence of a spike on pre- and post-synaptic terminal. Moreover, $a_{1}^{\text {pre }}$ and $a_{1}^{\text {post }}$ represent the non-Hebbian effect on synaptic weight resulting due to the occurrence of preand post-synaptic spike, respectively. $a_{2}^{\text {pre,post }}\left(t^{\prime}\right)$ governs the weight change that depends on the time difference between the current pre-synaptic spike and the last post-synaptic spike occurrence. This factor is responsible for reducing the weight according to Hebbian learning. On the other hand, if a postsynaptic spike occurs at a certain time instant, the time since last pre-synaptic spike arrival dictates strengthening of the synapse with the factor $a_{2}^{\text {post,pre }}\left(t^{\prime}\right) \cdot a_{2}^{\text {pre,post }}\left(t^{\prime}\right)$ and $a_{2}^{\text {post,pre }}\left(t^{\prime}\right)$ together describe a learning window $W\left(t^{\prime}\right)$, i.e., a rule for updating synaptic weights, as follows [9],

$$
W\left(t^{\prime}\right)= \begin{cases}a_{2}^{\text {post,pre }}\left(t^{\prime}\right)=A_{+} \exp \left(t^{\prime} / \tau_{1}\right), & \text { if } t^{\prime}<0 \\ a_{2}^{\text {pre,post }}\left(t^{\prime}\right)=A_{-} \exp \left(-t^{\prime} / \tau_{2}\right), & \text { if } t^{\prime}>0\end{cases}
$$

where $t^{\prime}<0$ shows the arrival of pre-synaptic spike before post-synaptic spike and $t^{\prime}>0$ shows otherwise.

\section{Performance Evaluation}

In this section, we evaluate the neuro-spike communication channel considering $M$ pre-synaptic neurons making only one synapse each with the output neuron as shown in Fig. 1. We utilize parameters given in Table I and simulate three different scenarios defined as follows for 1000 time steps:

- When there is no STDP, thus, synaptic weights are constant at $w_{\text {rest }}$.

- In the presence of STDP with no correlation among pre-synaptic spike trains generated by Poisson processes with the same rate, i.e., $\lambda_{i}=\lambda$.

- In the presence of STDP with correlated inputs. 
TABLE I. Simulation parameters.

\begin{tabular}{|l|c|l|}
\hline Parameters & Symbols & Values \\
\hline Normalized EPSP amplitude & $h_{i} w_{\text {rest }}$ & $1 \mathrm{mV}[5]$ \\
\hline Initial synaptic weight & $w_{\text {rest }}$ & 0.5 \\
\hline Time to reach EPSP peak & $t_{p}$ & $0.1 \mathrm{~ms}[7]$ \\
\hline Number of available vesicles & $N_{i}$ for all $i$ & $10[14]$ \\
\hline Resting potential & $v_{\text {rest }}$ & $-65 \mathrm{mV}$ \\
\hline Noise standard deviation & $\sigma_{n}$ & $0.1 \mathrm{mV}[5]$ \\
\hline Refractory period & $\tau_{r e f}$ & $2 \mathrm{~ms}[11]$ \\
\hline Number of inputs & $M$ & 300 \\
\hline \multirow{3}{*}{ STDP parameters } & $a_{0}, a_{1}^{\text {pre }}, a_{1}^{\text {post }}$ & $-1,0.01,0$ \\
\cline { 2 - 3 } & $A_{+}, A_{-}$ & $0.24,-0.1$ \\
\cline { 2 - 3 } & $\tau_{1}, \tau_{2}$ & $12.2,13.6 \mathrm{~ms}$ \\
\hline
\end{tabular}

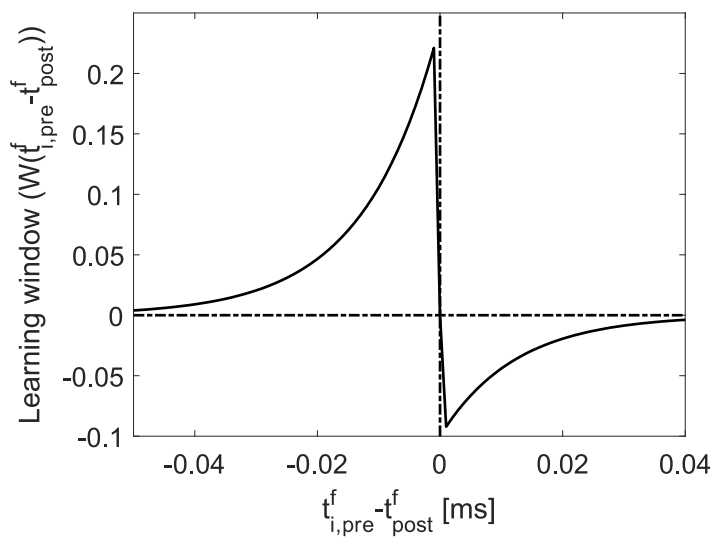

Fig. 2. Learning window.

Changes in synaptic efficacy using STDP model are defined using (3) and (4) and the learning window is shown in Fig. 2. To induce realistic synaptic plasticity, we need to ensure that synaptic weights remain in certain bounds. Thus, we consider initial values of $w_{i}(0)=w_{\text {rest }}$ and restrict the synaptic weights in the interval [0,1] according to [15]. Moreover, the parameters of STDP model are selected according to studies fitting the STDP model to experimental data [16].

\section{A. Correlation among Inputs and Synaptic Strength}

For introducing correlation among the pre-synaptic spike trains, we divide inputs into five groups $\left\{G_{1}, G_{2}, G_{3}, G_{4}, G_{5}\right\}$, where $G_{1}$ to $G_{4}$ have ten members each and the remaining neurons are in $G_{5}$. Let $T_{i} \sim \operatorname{Poiss}\left(\lambda_{i}\right)$, where $\lambda_{i}=\lambda$ for $i \in[1, M]$, be independent random variables. For inputs assigned to $G_{l}$ with $l \in[1,4]$ the spike trains are defined as $S_{i}=$ $T_{i}+C_{l}$, where $i \epsilon G_{l}$ and $C_{l} \sim$ Poiss $\left(\lambda_{C_{l}}\right)$. Hence, the correlation coefficient among any two neurons in these groups is calculated as,

$$
\rho_{i j}=\operatorname{corr}\left(S_{i}, S_{j}\right)=\left\{\begin{array}{ll}
\frac{\lambda_{C l}}{\lambda+\lambda_{C l}} & \text { if } i \neq j \\
1 & \text { if } i=j
\end{array},\right.
$$

where $i, j \in G_{l}$ and $l \epsilon[1,4]$. We select $\lambda_{c 1}=20 \mathrm{~Hz}, \lambda_{c 2}=50$ $\mathrm{Hz}, \lambda_{c 3}=70 \mathrm{~Hz}$ and $\lambda_{c 4}=100 \mathrm{~Hz}$. For neurons in $G_{5}$ the spike trains are defined as $S_{i}=T_{i}$. Note that spike trains of neurons assigned to different groups are independent.

The change in synaptic weights over a period of time is calculated by (3) and the ratio of strengthened or potentiated synapses in each group at the end of simulation time are plotted in Fig. 3 for different values of spiking threshold $\theta$. As shown in Fig. 3, the ratio of potentiated users, $R_{l}$, increases as the correlation among the users in the same group increases. However, according to (5), the correlation factor reduces with the increase in $\lambda$, thus, the number of potentiated inputs for different groups approach the uncorrelated scenario.

As $\lambda$ increases, the probability of input spike increases, however, moderate values of $\lambda$ are unable to generate enough number of post-synaptic spikes. Hence, the negative effect of $a_{2}^{\text {pre,post }}\left(t^{\prime}\right)$ is dominant compared to the positive impact of $a_{2}^{\text {post,pre }}\left(t^{\prime}\right)$ on synaptic strength. Thus, a drop in the value of $R_{l}$ is observed. Further increase in $\lambda$ generates more postsynaptic spikes increasing the effect of $a_{2}^{\text {post,pre }}\left(t^{\prime}\right)$, thus, improving the potentiation of the synapses.

When spiking threshold, $\theta$, increases more pre-synaptic spikes, thus, higher value of $\lambda$, is required to generate a postsynaptic spike. Hence, the drop in $R_{l}$ is shifting to the right from moderate towards higher values of $\lambda$ as shown in Fig. 3. Moreover, the value of $R_{l}$ at its drop is decreasing by increasing $\theta$ since the negative impact of $a_{2}^{\text {pre,post }}\left(t^{\prime}\right)$ is stronger because of existence of more pre-synaptic spikes.

\section{B. Probability of Spike Generation at Output Neuron}

The synaptic weights, $w_{i}(t)$, have a direct impact on the amplitude of EPSP as given by (1). Thus, change in $w_{i}(t)$ directly affects the probability of spike generation on postsynaptic terminal. As depicted in Fig. 3(a), more than 50\% of input neurons have synaptic weights greater than $w_{\text {rest }}$ for almost all values of $\lambda$. Hence, spike generation probability is higher in the neuro-spike communication channel with STDP at this spiking threshold as depicted in Fig. 4(a). Moreover, since the synapses for correlated inputs are strengthened more than uncorrelated ones, the probability of spike generation is also higher for the system with correlated inputs. Same pattern is observed for low values of $\lambda$ at $\theta=20 \mathrm{mV}$ and $\theta=25$ $\mathrm{mV}$ in Fig. 4(b-c). However, as shown in Fig. 3(b-c), more than $50 \%$ of the synapses are depressed for moderate values of $\lambda$ in the system with STDP at these spiking thresholds. Thus, the probability of output spike generation in this system is less than the system without STDP for moderate values of $\lambda$. As shown in Fig. 4(b-c), the system with STDP again outperforms the system without STDP for higher values of $\lambda$ since the percentage of potentiated synapses increases.

\section{CONCLUSION}

In this paper, we studied the impact of STDP on the performance of multiple access neuro-spike communication channel for correlated as well as uncorrelated input spike trains. As observed in the simulation results, correlation among inputs improves the probability of signal transmission over the channel as a result of strengthening the synapses of the 


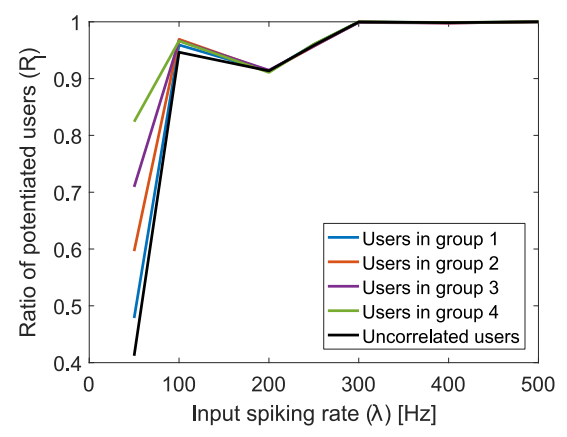

(a) $\theta=15 \mathrm{mV}$

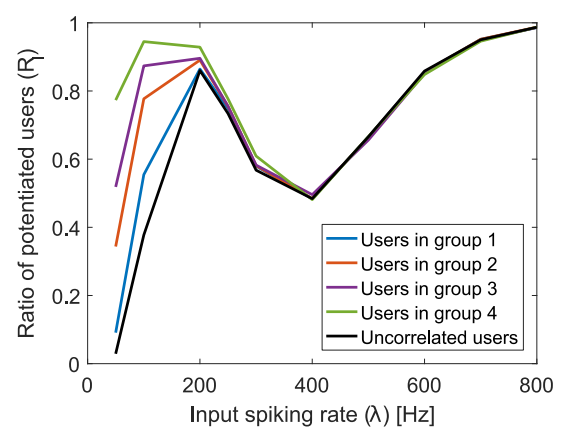

(b) $\theta=20 \mathrm{mV}$

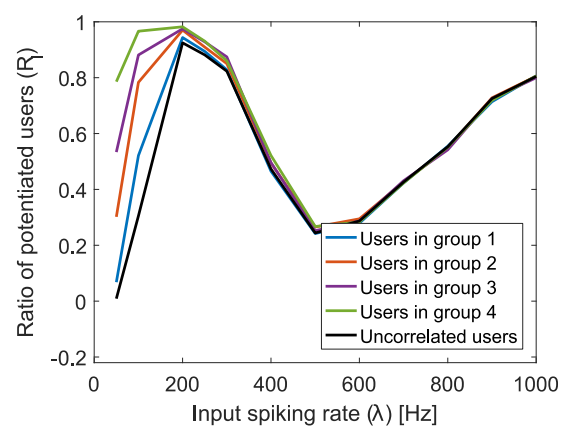

(c) $\theta=25 \mathrm{mV}$

Fig. 3. Ratio of synaptic weights greater than $w_{\text {rest }}$, i.e., potentiated synapses, for different spiking thresholds.

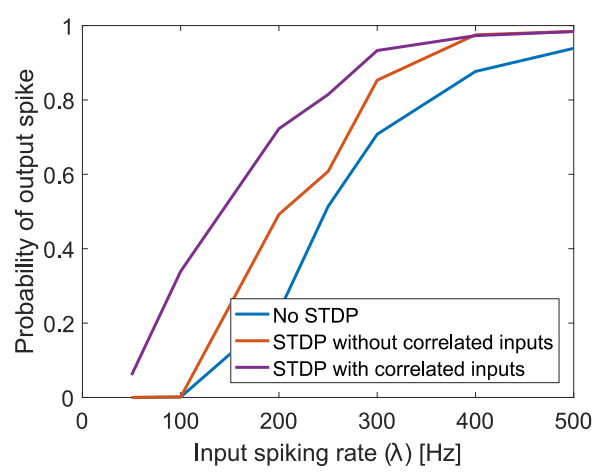

(a) $\theta=15 \mathrm{mV}$

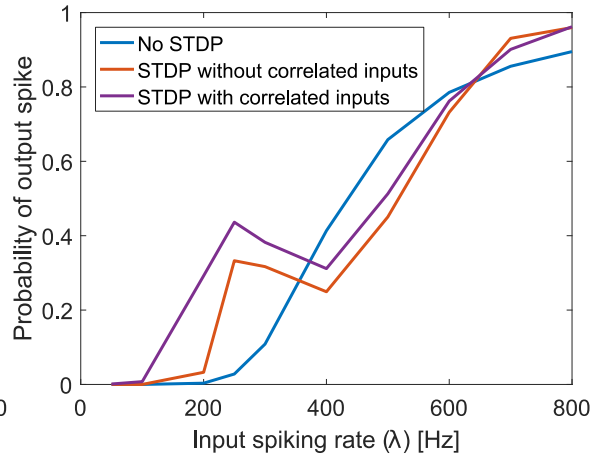

(b) $\theta=20 \mathrm{mV}$

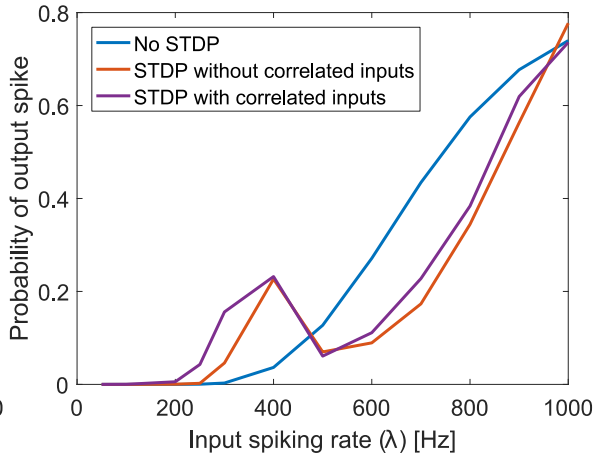

(c) $\theta=25 \mathrm{mV}$

Fig. 4. Probability of spike generation in output for different spiking thresholds.

correlated inputs. Furthermore, it is observed that the changes in the spiking threshold, which happens in real scenario, have nonlinear impacts on the probability of signal transmission over this communication channel. Thus, our analysis signifies the importance of a comprehensive model for neuro-spike communication channel consisting of long-term plasticity to fully evaluate the performance of the nanonetwork using neurospike communication paradigm as well as for getting insights into the functionality of the brain.

\section{ACKNOWLEDGMENT}

This work was supported in part by the ERC projects MINERVA (ERC-2013-CoG \#616922) and the ERC Proof of Concept project MINRGRACE (ERC-2017-PoC \#780645).

\section{REFERENCES}

[1] A. Citri and R. C. Malenka, "Synaptic plasticity: multiple forms, functions, and mechanisms," Neuropsychopharmacology, vol. 33, no. 1, p. 18, 2008.

[2] G. W. Crabtree and J. A. Gogos, "Synaptic plasticity, neural circuits, and the emerging role of altered short-term information processing in schizophrenia," Frontiers in synaptic neuroscience, vol. 6, p. 28, 2014.

[3] T. Bliss et al., "Synaptic plasticity in health and disease: introduction and overview," 2014.

[4] H. Ramezani and O. B. Akan, "Information capacity of vesicle release in neuro-spike communication," IEEE Communications Letters, 2017.

[5] D. Malak and O. B. Akan, "A communication theoretical analysis of synaptic multiple-access channel in hippocampal-cortical neurons," IEEE Trans. Commun., vol. 61, no. 6, pp. 2457-2467, 2013.
[6] M. Veletic et al., "Peer-to-peer communication in neuronal nanonetwork," IEEE Trans. Commun., vol. 64, no. 3, pp. 1153-1166, 2016.

[7] T. Khan et al., "Diffusion-based model for synaptic molecular communication channel," IEEE Trans. NanoBioscience, 2017.

[8] H. Ramezani et al., "Information Theoretical Analysis of Synaptic Communication for Nanonetworks," IEEE INFOCOM Conference, 2018.

[9] W. Gerstner and W. M. Kistler, Spiking neuron models: Single neurons, populations, plasticity. Cambridge university press, 2002.

[10] M. C. van Rossum and G. G. Turrigiano, "Correlation based learning from spike timing dependent plasticity," Neurocomputing, vol. 38, pp. 409415, 2001

[11] R. Kobayashi et al., "Made-to-order spiking neuron model equipped with a multi-timescale adaptive threshold," Front. in comp. neuroscience, vol. 3, p. $9,2009$.

[12] L. E. Dobrunz and C. F. Stevens, "Heterogeneity of release probability, facilitation, and depletion at central synapses," Neuron, vol. 18, no. 6, pp. 995-1008, 1997.

[13] D. E. Feldman, "The spike-timing dependence of plasticity," Neuron, vol. 75 , no. 4, pp. 556-571, 2012.

[14] T. Schikorski and C. F. Stevens, "Quantitative ultrastructural analysis of hippocampal excitatory synapses," J. Neurosci., vol. 17, no. 15, pp. $5858-5867,1997$.

[15] W. M. Kistler and J. L. v. Hemmen, "Modeling synaptic plasticity in conjunction with the timing of pre-and post-synaptic action potentials," Neural Computation, vol. 12, no. 2, pp. 385-405, 2000.

[16] H. Hayashi and J. Igarashi, "Ltd windows of the stdp learning rule and synaptic connections having a large transmission delay enable robust sequence learning amid background noise," Cognitive Neurodynamics, vol. 3, no. 2, pp. 119-130, 2009. 